\title{
ORALIDISEASES
}

\section{Investigating the relationship between hyposalivation and mucosal wetness}

\begin{tabular}{|c|c|}
\hline Journal: & Oral Diseases \\
\hline Manuscript ID: & ODI-08-09-OM-1372.R3 \\
\hline Manuscript Type: & Original Manuscript \\
\hline $\begin{array}{r}\text { Date Submitted by the } \\
\text { Author: }\end{array}$ & 01-Apr-2010 \\
\hline Complete List of Authors: & $\begin{array}{l}\text { Osailan, Samira; King's Collgege London, Salivary Research Unit } \\
\text { Pramanik, Rashida; King's College London, Salivary Research Unit } \\
\text { Shirodaria, Soha; GlaxosmithKline, Consumer Health Care } \\
\text { Challacombe, Stephen J; King's College London Dental Institute at } \\
\text { Guy's, King's and St Thomas Hospital, Department of Oral Medicine } \\
\text { Proctor, Gordon; Kings College London Dental Institute, Salivary } \\
\text { Research Unit }\end{array}$ \\
\hline Keywords: & salivary gland disease, Diagnostics, Pathology \\
\hline
\end{tabular}



Investigating the relationship between hyposalivation and mucosal
wetness

\author{
Authors: \\ Samira Osailan ${ }^{1, *}$ Rashida Pramanik ${ }^{1}$, Soha Shirodaria ${ }^{2}$, Stephen.J. \\ Challacombe $^{1}$ and Gordon B. Proctor ${ }^{*}$ \\ ${ }^{1}$ King's College London Dental Institute, London, ${ }^{2}$ GlaxoSmithKline Consumer \\ Healthcare, Weybridge, United Kingdom. \\ Running title: mucosal wetness can measure oral dryness
}

Corresponding authors:

*Samira Osailan \& *Gordon Proctor

Salivary Research Unit,

Floor 17, Tower Wing

King's College London Dental Institute, London SE1 9RT

UK

Tel: +44 (0) 2071887461

Fax: +44 (0) 2071887458

E-mail: samira.osailan@kcl.ac.uk

Email: gordon.proctor@kcl.ac.uk 


\section{Authors: \\ S. Osailan ${ }^{1,{ }^{*}}$ R. Pramanik ${ }^{1}$, S. Shirodaria ${ }^{2}$, S.J. Challacombe ${ }^{1}$ and G. B. Proctor ${ }^{1 *}$ Title: Investigating the relationship between hyposalivation and mucosal wetness Oral Diseases}

\section{ABSTRACT}

Mucosal wetness (MW) reflects the layer of residual saliva that covers the oral mucosal surfaces. Objectives: to determine MW at different oral mucosa sites and to investigate the relationship between MW, Unstimulated Whole salivary flow rates (UWS) and clinical oral dryness score (CODS). Method: 100 dry mouth patients and 50 healthy subjects. MW was sampled with filter paper strips at four sites inside the mouth; Anterior Hard Palate (AHP), Buccal mucosa (BUC), Anterior Tongue (AT), Lower Lip (LL) and measured with a micromoisture meter. Reproducibility was assessed by repeated sampling and diurnal variation was examined. Results: MW in healthy subjects showed site variation and means \pm SD were; AHP $(11 \pm 11.7 \mu \mathrm{m})$, BUC $(32 \pm 14.8 \mu \mathrm{m})$, AT $(65 \pm 17.2$ $\mu \mathrm{m})$, and LL $(25 \pm 13.5 \mu \mathrm{m})$. Dry mouth patients with reduced UWS showed increased CODS. MW at all four sites was significantly reduced $(p<0.05)$ in dry mouth patients compared with the healthy subjects. Reproducibility of MW measurement using the intra-class correlation coefficient showed agreement at different visits within subject. MW of the AT showed a positive correlation with UWS $(p<0.05)$. Conclusion: MW is a reliable measure of oral dryness and had a positive correlation with UWS.

Key words: xerostomia, mucosal wetness, salivary secretion.

\section{Corresponding authors:}

Dr Samira Osailan \& Prof Gordon Proctor

Salivary Research Unit, Floor 17, Tower Wing 
King's College London Dental Institute, London SE1 9RT, United Kingdom

Fax: +44 (0) 2071887458

E-mail: samira.osailan@kcl.ac.uk or samira_osailan@yahoo.co.uk Email: gordon.proctor@kcl.ac.uk 


\section{INTRODUCTION}

Dry mouth is most commonly caused by alterations in salivary gland function, dehydration, and cognitive alteration. Anxiety or depression and stress can be a cause of both subjective (xerostomia) (Fox et al., 1985) and objective (hyposalivation) feelings of dry mouth (Bergdahl \& Bergdahl, 2000). It is known that drugs are the most common cause of the dry mouth condition and complaints of xerostomia are a frequent side effect of many drugs (reviewed by Scully, 2003). Hyposalivation is especially known in those drugs used to treat anxiety, depression, and stress but is usually reversible. Salivary gland diseases associated with hyposalivation include primary or secondary Sjögren's syndrome, (Sjögren, 1933; Navazesh et al., 1996; Price \& Venables, 2002; Kassan \& Moutsopoulos, 2004; Atkinson et al., 2005), and Sialadenitis, Nodal Osteoarthiritis, Xerostomia syndrome (SNOX; Kassimos et al., 1995). Sjögren's syndrome affects approximately $0.4 \%$ of the population and has a male: female ratio of 1: 10 (Fox 2005). Other conditions and systemic diseases where dry mouth could be a relevant complaint include diabetes, thyroid disorders, connective tissue diseases and graft versus host disease (Atkinson et al., 1994; Scully, 2003). The prevalence of xerostomia in the general population ranges between 10-20\% in different published studies (Fox et al., 1985; Pujol, 1998). Prevalence is greater in females and increases with increased medication (Nederfors, 1997; Schein et al., 1999). In the elderly (60+ years) population prevalence is approximately $20 \%$ (Ben-Aryeh et al., 1985; Nederfors et al., 1997; Nayak et al., 2004).

As with most symptoms, it has been difficult to quantify dry mouth complaints precisely and reproducibly. Investigators have used a variety of methods to assess oral dryness including: questionnaires, Visual Analog-Scales (VAS), simple functional measures such as observing if a tongue blade adheres to the buccal mucosa or if a patient can chew and swallow dried biscuits without water (Fox, 2005). Dry mouth can also be assessed by measuring the volume of residual saliva on mucosal surfaces using filter paper and micro-moisture meter 
and calculating thickness (DiSabato-Mordarski \& Kleinberg, 1996; Won et al., 2001; Lee et al. , 2002; and Eliasson et al. , 2005) and more recently mucosal wetness devices have been used (Kakinoki et al., 2004; Takahashi et al., 2005).

Collins \& Dawes (1987) calculated the average surface area of the mouth to be $214.7 \mathrm{~cm}^{2}$ and calculated the thickness of the salivary film in the mouth to be 44 $\mu \mathrm{m}$, by dividing the mean residual saliva in the mouth by surface area. The thickness of the salivary film is governed it part by the rheological properties of saliva. It is apparent that the thickness and composition of the salivary film will vary in different parts of the mouth depending upon the position in relation to salivary glands.

The current study aims are to determine the normal variation of Mucosal Wetness (MW) at different oral mucosa sites and secondly to determine the relationship between mucosal wetness, unstimulated whole mouth (UWS) salivary flow rate, Clinical Oral Dryness Score (CODS). 


\section{MATERIAL \& METHOD}

\subsection{Study subjects}

Samples were collected from a total of 100 patients with a mean age of $62 \pm 11$ years (range 22-82 years) attending Oral Medicine clinics at Guy's Hospital. They all complained of dry mouth and were divided into five groups according to their diagnosis: primary and secondary Sjögren's syndrome (SS1 \& SS2); Drug induced Hyposalivation (DIH); non-Sjögren's but presence of sialadenitis, nodular osteoarthritis, xerostomia (SNOX); none of the above (NOS).

Fifty healthy age matched subjects who did not complain of dry mouth were selected as controls and had a mean age of $60 \pm 15$ years (range 22-83). They were recruited from members of staff and from a residential home for the elderly. All patients and participants were given an explanation and information sheet of the study and all gave their informed consent prior to the procedure. The study was performed under ethical approval of Guy's \& St Thomas' Hospitals (Local) Research Committee. Ten healthy volunteers with mean age \pm SD is $35 \pm 9.5$ years $(n=10)$ from the 50 controls were used to validate the reproducibility of mucosal wetness measurements.

\subsection{Assessment of patients and collection of samples}

\section{a. Clinical oral dryness score}

The signs of dryness in the mouth were examined using a scoring system (the Clinical Oral Dryness Score, CODS) which is composed of ten features: 1) Mirror sticks to buccal mucosa, 2) Mirror sticks to tongue, 3) Saliva frothy, 4) No saliva pooling in floor of mouth, 5) Tongue shows loss of papillae, 6) Altered gingival architecture/ smooth (especially anterior), 7) Glassy appearance to oral mucosa (especially palate), 8) Tongue lobulated / deeply fissured, 9) Cervical caries (more than two teeth), 10) Mucosal debris on palate (excluding under dentures). This technique was validated and the data presented elsewhere (Challacombe et al, 2008). 


\section{b. Unstimulated whole mouth (UWS) salivary flow}

UWS was measured for 10 minutes and the subject was asked to spit into a preweighed vessel and not to swallow any saliva. UWS flow rate was calculated and expressed $\mathrm{ml} / \mathrm{min}$, taking $1 \mathrm{~g}$ saliva $=1 \mathrm{ml}$.

\section{c. Mucosal wetness (MW) measurements}

The thickness of residual saliva (oral mucosal wetness) was measured in dry mouth patients $(n=100)$ and aged matched healthy subjects $(n=50)$ using a filter paper strip (Oraflow Inc, USA) and micro-moisture meter (Periotron® 8000; Oraflow Inc, USA). A filter paper strip with a diameter $7.5 \mathrm{~mm}$ covering an area of $44 \mathrm{~mm}^{2}$ was placed immediately on the mucosa after swallowing and was gently pressed flat with a finger of a gloved hand. After $10 \mathrm{sec}$ the paper strip was transferred to the sensors of the micro-moisture meter. Four mucosal sites were measured; Anterior Hard palate (AHP), Buccal (BUC), Anterior Tongue (AT), and Lower Lip (LL) (Figure 1). A calibration curve previously constructed using volumes of UWS saliva was used to calculate the volumes $(\mu \mathrm{l})$ of residual saliva collected from mucosal surfaces and then mucosal thickness $(\mu \mathrm{m})$ was calculated. For the validation of mucosal wetness measurements 10 volunteer subjects were assessed over 10 visits, 5 morning (9-12 am) and 5 afternoon (2-5 pm) visits. UWS was also measured on each occasion.

\subsection{Statistics}

Statistical analysis was carried out using SPSS computer software version 15. An analysis of variance (ANOVA) and intra-class correlation coefficient (ICC) were used in order to validate the mucosal wetness measurement between and within subjects. For the purpose of analysis, dry mouth patients' data was grouped according to either diagnostic or to UWS flow rate groups. All groups were compared with age matched controls. Correlations between MW and UWS salivary flow rate were determined using Pearson (parametric) correlation analysis. 


\section{RESULTS}

\subsection{Validation of mucosal wetness measurement}

Measurement of MW using filter paper strips and the micro-moisture meter showed good reproducibility. Intra-class correlation coefficients (ICC) for MW measurements from the same subjects $(n=10)$ at different visits for AHP, BUC, AT and LL were $0.49,0.48,0.58,0.53$ respectively ( $P<0.02$ for all surfaces). No significant difference was found between morning and afternoon for oral mucosal wetness values for AHP, BUC, AT, and LL (Figure 2) and UWS salivary flow rate.

\subsection{Mucosal wetness of dry mouth patients and controls}

In dry mouth patients, the means \pm SD MW of all four surfaces were significantly reduced by approximately 50\% compared with age-matched controls (Figure 3 ) but the trend was the same. That is AHP had the thinnest and AT tongue had the thickest MW amongst both patients and controls (Figure 3 ).

\subsection{Correlation between UWS salivary flow and mucosal wetness}

Overall UWS was significantly $(p<0.05)$ directly correlated with MW at all four sites. Pearson correlation coefficients for each site were $r=0.22(\mathrm{AHP}), r=0.18$ (BUC), $r=0.4(A T), r=0.3(\mathrm{LL})$ respectively.

Subjects were grouped according to UWS salivary flow rate as follows: three patient groups with low flow $(0-0.1 \mathrm{ml} / \mathrm{min}, \mathrm{n}=57)$, moderate flow $(0.1-0.2$ $\mathrm{ml} / \mathrm{min}, \mathrm{n}=25$ ), high flow $(>0.2 \mathrm{ml} / \mathrm{min}, \mathrm{n}=18$ ) and a fourth group of controls (mean flow $=0.45 \mathrm{ml} / \mathrm{min}$, range $0.2-1.0 \mathrm{ml} / \mathrm{min}, \mathrm{n}=50)$. The group with lowest flow $(<$ $0.1 \mathrm{ml} / \mathrm{min}$ ) showed a significant $(p<0.05)$ reduction in MW at all four sites (AHP, BUC, AT and LL) compared with controls (Figure 4a). The AHP, BUC and LL mucosal surfaces showed no significant differences between the low and high UWS patient groups whilst AT showed a significant $(p<0.05)$ reduction in MW between all UWS flow rate patient groups.

In addition, when a patient group $(\mathrm{n}=14)$ with UWS flow rate $>0.2-0.3 \mathrm{ml} / \mathrm{min}$ with a mean $\pm \mathrm{SD}(0.24 \pm 0.01 \mathrm{ml} / \mathrm{min})$ was compared with a similar UWS flow 
rate $($ mean $=0.26 \pm 0.01 \mathrm{ml} / \mathrm{min}, \mathrm{n}=10$ ) control group there was a significant $(p<$ 0.05 ) reduction of MW at BUC and AT surfaces (Figure $4 \mathrm{~b}$ ). The AHP showed no difference in wetness whilst the LL showed a significant difference only by a one-tailed t-test $(p<0.05)$. The different flow rate groups of control subjects did not show any statistically significant differences in MW of different oral surfaces.

\subsection{The relationship between CODS and UWS salivary flow rate}

There was inverse correlation between CODS and UWS salivary flow rate of dry mouth patients and healthy subjects (aged matched controls). Even in dry mouth patients with low CODS $(1-3)$ has a significant $(p<0.01)$ reduction in there UWS compared with the controls (Figure 5). 


\section{DISCUSSION}

The findings show that the mucosal wetness differ on oral surfaces; AHP, BUC, AT and LL. The trend of MW follows the same trend in healthy subjects as well as dry mouth patients. In both groups the wettest surface was AT, followed by BUC, LL then AHP. Our findings are similar to those in previous studies (DiSabato-Mordarski \& Kleinberg, 1996; Won et al., 2001; Lee et al., 2002). For AHP the values of at least $10 \mu \mathrm{m}$ on the palate appeared necessary to avoid complaints of dry mouth (Wolff and Kleinberg, 1998). Previous studies suggest that the percentage contribution of different glandular salivas to the total saliva on different oral surfaces varies. Thus the percentage contribution of parotid saliva to the total saliva on different oral surfaces is not the same (Sas \& Dawes, 1997). For example, the surface vestibular to the upper right molars appears to have a $50-60 \%$ contribution from parotid saliva whilst the surfaces vestibular or lingual to the lower incisors have only a $5-7 \%$ contribution. These differences in composition along with differing densities of minor salivary glands in the submucosae of oral surfaces will also presumably contribute in determining the wetness of the different surfaces measured in the present study.

Measurement of MW by filter paper sampling and micro-moisture meter measurement showed good reproducibility and consistency at all four sites. AT and BUC surfaces were very consistent sites while anterior hard palate showed variations between individuals. Previously it has been reported that unstimulated whole salivary flow rates show a circadian rhythm (Dawes, 1972). However, in the present study, neither mucosal wetness nor UWS flow rate showed a significant difference between samples taken in the morning (9-12) or in the afternoon (2-5). This suggests that mucosal wetness and UWS flow rate can be measured during the hours of normal clinics.

In dry mouth patients, MW at four sites (AHP, BUC, AT, LL) was significantly reduced by approximately $50 \%$ compared with controls. Other studies on 
subjects with oral dryness have reported similar findings (Wolff \& Kleinberg, 1998; Won et al., 2001; Lee et al., 2002; Eliasson et al., 2005). In addition, the distribution pattern of the MW on the four mucosal surfaces was the same in patients and controls. i.e., the AT had the thickest and anterior AHP had the thinnest layers of MW which is in agreement with Lee et al, (2002). Whilst Wolff and Kleinberg (1998) found that the posterior tongue had the thickest layer of mucosal wetness. Although there appeared to be a decrease in wetness of the AHP in the dry mouth patients compared to controls there was more variation between individuals with means of $7 \pm 7.2 \mu \mathrm{m}$ and $11 \pm 11.3 \mu \mathrm{m}$ for patients and controls respectively. In the present study all of the patient groups showed a mean $\mathrm{MW}$ of $<10 \mu \mathrm{m}$ but the normal control group showed a mean thickness of only $11 \mu \mathrm{m}$. Others have shown that there was no significant difference in palatal saliva secretion between Sjögren's syndrome patients and healthy control (Marton et al, 2004).

Oral dryness assessed using CODS was significantly increased in all patients complaining of dry mouth. Subjects with lower UWS salivary flow rates showed the highest CODS values. Since mucosal wetness of all surfaces (AHP, AT, BUC, LL) showed a significant decrease with a reduction in UWS salivary flow rate it can be inferred that CODS and mucosal wetness also show an inverse relationship. Thus reduced $\mathrm{MW}$ is linked with increased CODS and clinical features of oral dryness. A positive correlation between MW and UWS salivary flow rate has also been previously reported (Wolff \& Kleinberg 1998). The anterior tongue showed a different pattern to the other surfaces with a 'stepped' decrease in wetness that mirrored the decrease in UWS flow rate. An explanation for this is that the tongue is the mobile part in the mouth and its fluid coating is derived from all contributions to the whole mouth saliva volume.

Patients with higher UWS flow rates $(>0.2-0.3 \mathrm{ml} / \mathrm{min})$ still showed a significant reduction in $\mathrm{MW}$ of the BUC and $\mathrm{AT}$ surfaces compared to controls with similar UWS flow rates. Others have observed a decrease in labial mucosal wetness in 
subjects with a subjective complaint of dry mouth (Eliasson et al 1996; Niedermeier et al 1989; Shern et al 1990). Therefore measuring mucosal wetness is an important investigation in the management of dry mouth patients since it is a direct measure of wetness that can discriminate between normal subjects and dry mouth patients. Our findings suggested that the reduction in mucosal wetness could be an early sign of dry mouth observed before UWS flow rate is obviously reduced. However, this needs to be substantiated on a larger numbers of samples. It may be that this group of patients (UWS $>0.2-0.3$ $\mathrm{ml} / \mathrm{min}$ ) had a more than $50 \%$ reduction in their baseline UWS salivary flow rate and consequently had reduced mucosal wetness. It has been reported that a subject needs at least a $50 \%$ reduction in baseline resting (unstimulated) salivary flow rate before dry mouth is experienced and this may coincide with a decrease in oral mucosal wetness (Dawes, 1987; Wolff and Kleinberg, 1999). It may also be that the patients with higher UWS flows but reduced mucosal wetness have saliva with altered mucosal coating properties due to changes in composition. There is evidence of reduced mucin sulphation in Sjögren's syndrome and this may impact on surface coating properties or water retention (Allende et al., 2008). Changed composition could result from a relatively greater reduction in submandibular secretion which might reduce mucin levels in whole mouth saliva, although results from a previous study do not support this idea (van den Berg et al., 2007). It would be interesting to examine the rheological and wetting properties and mucin content of salivas from such patients. When control subjects were divided into UWS flow rate groupings it is evident that there was little difference in wetness of the oral surfaces with increase UWS flow rate. It can be suggested that above a UWS flow rate of $0.2 \mathrm{ml} / \mathrm{min}$ there is no further significant retention of residual fluid on oral surfaces.

In conclusion, mucosal wetness can potentially be used as an index of oral dryness. It is a reliable, simple method which can be used at the chair side to measure oral dryness. There is a positive correlation between oral mucosal wetness and unstimulated salivary flow rate. 


\section{ACKNOWLEDGMENTS}

This study was funded by GlaxoSmithKline Consumer Health Care, Weybridge, Surrey, United Kingdom. Financial support was also received from the Department of Health via the National Institute for Health Research (NIHR) comprehensive Biomedical Research Centre award to Guy's \& St Thomas' NHS Foundation Trust in partnership with King's College London. 


\section{REFERENCES}

- Alliende C, Kwon YJ, Brito M et al. Reduced sulfation of MUC5b is linked to xerostomia in patients with Sjögren's syndrome. Ann. Rheum. Dis. 2008; 67: (10):1480-1487.

- Atkinson J.C., Wu A.J. Salivary gland dysfunction: causes, symptoms, treatment. J. Am. Dent. Assoc. 1994; 125:409-416.

- Atkinson J C, Grisius M and Massey W. Salivary hypofunction and xerostomia: diagnosis and treatment. Dent. Clin. North Am. 2005; 49 (2): 309-26.

- Ben-Aryeh H., Miron D., Berdicevsky I, Szargel R., Gutman D. xerostomia in elderly: prevalence, diagnosis, complications and treatment. Gerodontology $1985 ; 4: 77-82$.

- Bergdahl M and Bergdahl J. Low unstimulated flow and subjective oral dryness: association with medication, anxiety, depression and stress. J. Dent. Res 2000; 79: 1652-1658.

- Challacombe S.J. ,Osailan S.M., Pramanik R., Shirodaria S., Urquhart D., Mason S.C. and Proctor G.B. A Clinical Score of oral Dryness: relation to salivary flow rates J. Dent. Res. 2008.

http://iadr.confex.com/iadr/2008Toronto/techprogram/abstract_106439.htm.

- Carpenter G.H., Proctor G.B., Pankhurst C.L., O'Donohue J., Scott D., Hunnable M.P. Sialochemical markers of salivary gland involvement with Sjögren's syndrome secondary to rheumatoid arthritis and primary biliary cirrhosis. J. Oral. Pathol. Med. 2000; 29 (9):452-9. 
- Collins L.M.C. and Dawes C. The surface area of the adult human mouth and thickness of salivary film covering the teeth and oral mucosa. J. Dent. Res. 1987; 66 (8): 1300-02.

- Dawes, C. Circadian rhythms in human salivary flow rate and composition. $J$ Physiol. 1972; 220 (3):529-545.

- Dawes C. Physiological factors affecting salivary flow rate, oral sugar clearance, and the sensation of dry mouth in man. J. Dent. Res.1987; 66: 64853.

- Disabato-Mordarski T. and Kleinberg I. Measurement and comparison of the residual saliva on various oral mucosae and dentition surfaces in humans. Arch oral Biol. 1996; 41(7): 655-65.

Eliasson L., Birkhed D., Heyden G., Strömberg N., Studies on human minor salivary gland secretions using the Periotron ${ }^{\circledR}$ method. Arch. oral Biol. 1996; 41: 1179-1182.

- Eliasson L., Almståhl A., Lingström P., Wikström M. and Carlén A. Minor gland saliva flow rate and proteins in subjects with hyposalivation due to Sjögren's syndrome and radiation therapy. Arch. oral Biol. 2005; 50: 293-9.

- Fox P.C., van der Ven P.F., Sonies B.C., Weiffenbach J.M., Baum B.J. Xerostomia: evaluation of a symptom with increasing significance. J. Am. Dent. Assoc. 1985; 110(4):519-25.

- Fox P. 2005 www.symptomresearch.nih.gov/chapter 27/index.htm in Symptom Research: 
- Kakinoki Y, Nakamura S, Koseki T. The practice of the test of saliva and the point of diagnosis. Dental outlook 2004; 103: 47-52.

- Kassan S.S., Moutsopoulos H.M., Clinical manifestations and early diagnosis of Sjögren's syndrome. Arch. Intern. Med. 2004; 164:1275-84.

- Kassimos D.G., Choy E.H.S, Challacombe S.J., Panayi G.S. The prevalence of xerostomia in a population with primary generalized osteoarthritis (PGOA). Br. J. Rheumatol. 1995; 34:132.

- Lee S.K., Lee S.W., Chung S.C., Kim Y.K. and Kho H. S. Analysis of residual saliva and minor salivary gland secretions in patients with dry mouth. Arch. oral Biol. 2002; 47: 637-41.

- Marton K, Boros I, Fejerdy P, Madlena M. Evaluation of Unstimulated flow rates of whole and palatal saliva in healthy patients wearing complete denture and in patients with Sjögren's syndrome. J. Prosthet. Dent. 2004; 91 (6):577-81.

- Navazesh M., Brightman V.J.,Pogoda J.M. Relation of medical status, medication, and salivary flow rates in adults of different age. Oral Surg. Oral Med. Oral Pathol. Oral Radiol. Endod. 1996; 81:172-6.

- Nayak L. Wolff A., Fedele S., Martin-Granizo R., Reichart P., Russo L., Mignogna M., Strietzel F. The Burden of Xerostomia in Independent Communitydwelling Older Adults: Results from the Saliwell Project. Oral Biosci. Med. 2004; 1(4): 283-89.

- Niedermeier, W., Hüber, M. Quantitative studies on the secretory output of the palatine salivary glands. Dtsch Zahnärztl Zschr 1989; 44: 37- 40. 
- Nederfors T, Isaksson R, Mornstad H, Dahlof C. Prevalence of perceived symptoms of dry mouth in an adult Swedish population--relation to age, sex and pharmacotherapy. Community Dent Oral Epidemiol. 1997; 25(3):211-6.

- Pujol T, Coma M, Pujol M, Postigo P. Prevalence of xerostomia in the general population. Aten. Primaria. 1998; 21(4):225-8.

- Price E.J., and Venables P.J.W. Dry eyes and mouth syndrome-a subgroup of patients presenting with sicca symptoms. Rheumatology 2002; 41: 416-422

- Sas R., Dawes C. The intra-oral distribution of unstimulated and chewing gum stimulated parotid saliva. Arch oral. Biol. 1997; 42: 469-474

- Scully C. Drug effects on salivary glands: dry mouth oral Dis. 2003; 9(4):165-76.

- Schein O.D., Hochberg M.C., Munoz B, Tielsch J. M., Bandeen-Roche K., Provost T, Anhalt G. J., West S. Dry Eye and Dry Mouth in the Elderly: A Population-Based Assessment. Arch. Intern. Med. 1999; 159:1359-1363.

- Shern R.J., Fox P.C., Cain J.L., and Li S-H. A method for measuring the flow of saliva from the minor salivary glands. J. Dent. Res. 1990,69(5):1146-1149.

- Sjögren H. Zur Kenntnis der Keratoconjunctivitis sicca (Keratitis filiformis bei Hypofunktion der Tränen-drusen). Acta Ophthalmol. 1933;11:1-151.

- Takahashi F, Koji T and Morita O. oral Dryness Examinations : Use of an oral Moisture Checking Device and a Modified Cotton Method. Prosthodontic. Res. Pract. 2005; 5: 26-30.

- Van den Berg I, Pijpe J, Vissink A. Salivary gland parameters and clinical data related to the underlying disorder in patients with persisting xerostomia. Eur. J. Oral Sci. 2007; 97-102

- Wolff M.S., Kleinberg I. oral mucosa wetness levels in hypo- and normosalivators. Arch. oral. Biol. 1998; 43: 455-62. 
- Wolff M, Kleinberg I: The effect of ammonium glycopyrrolate (Robinul®-induced xerostomia on oral mucosal wetness and flow of gingival crevicular fluid in humans. Arch. oral. Biol. 1999; 44:97-102.

- Won S.H., Kho H.S., Kim Y.K., Chung S.C., Lee S.W. Analysis of residual saliva and minor salivary gland secretions in patients with dry mouth. Arch. oral Biol. 2001; 46: 619-624. 


\section{Legends Figures and tables}

Figure 1. Periotron $® 8000$ micro-moisture meter, filter paper strip Sialopaper strip $^{\mathrm{TM}}$ and four mucosal wetness surfaces inside the mouth, (a) Anterior hard palate (AHP), (b) Buccal (BUC), (c) Anterior Tongue (AT), (d) Lower Lip (LL) surfaces on a healthy subjects.

Figure 2. Mean values of mucosal wetness in $\mu \mathrm{m}$ from four oral surfaces (AHP, BUC, AT, LL) were measured from 10 subjects in the morning (am, light bars)) and the afternoon ( $\mathrm{pm}$, dark bars) and 5 visits for each time point of the same subjects. It showed there are no significant $(P<0.05)$ differences in wetness of all the four surfaces between morning and afternoon.

Keys: $\mathrm{AHP}=$ Anterior Hard Palate, $\mathrm{BUC}=$ Buccal, $\mathrm{AT}=$ Anterior Tongue, $\mathrm{LL}=$ Lower Lip. Error bars represent SEM.

Figure 3. Mean values of mucosal wetness at four surfaces (AHP, BUC, AT, LL) from dry mouth patients ( $n=100$, dark bars) and healthy subjects as controls ( $n=50$, light bars). There is a significant $(p<0.05)$ reduction in wetness on all mucosal sites from dry mouth patients compared with controls.

Keys: $\mathrm{AHP}=$ Anterior Hard Palate, $\mathrm{BUC}=$ Buccal, $\mathrm{AT}=$ Anterior Tongue, $\mathrm{LL}=$ Lower lip. ${ }^{*}=$ statistically significant $(P<0.05)$. Error bars represent SEM

Figure 4. A relationship between mucosal wetness (MW) and unstimulated whole salivary flow rates (UWS) groups. (a) It shows 4 groups of UWS; 3 groups from dry mouth patients (3 groups; 0-0.1, > 0.1-0.2, > 0.2-0.3 ml/min) and controls (one group > 0.2-1.0 ml/min, $n=50$ ). All four sites; AHP, BUC, AT, LL in patients with different flow groups were significantly $(P<0.01)$ less than controls. AT shows a statistically significant $(P<0.05)$ increase in MW $(\mu \mathrm{m})$ with increase in UWS salivary flow. (b) The patient group (UWS $>0.2-0.3 \mathrm{ml} / \mathrm{min}$ ) with a mean of $0.24 \pm 0.01 \mathrm{ml} / \mathrm{min}$ had a significant reduction in $\mathrm{MW}$ at BUC $(p<0.001)$, AT $(p<0.05)$ and were the same at AHP and LL compared with controls of a similar 
(UWS > 0.2-0.3 ml/min) flow rate with a mean of $0.26 \pm 0.01 \mathrm{ml} / \mathrm{min}$. Keys: $\mathrm{AHP}=$ Anterior Hard Palate, $\mathrm{BUC}=$ Buccal, $\mathrm{AT}=$ Anterior Tongue, $\mathrm{LL}=$ Lower Lip. $1=$ statistically significant different between patients ( 3 groups) and controls. ${ }^{2}=$ statistically significant between the three flow rate $(0-0.1,>0.1-0.2,>0.2-0.3$ $\mathrm{ml} / \mathrm{min}$ ) patients groups at the AT site only. ${ }^{3}=$ BUC is statistically significant different between patient group $>0.2-0.3 \mathrm{ml} / \mathrm{min}$ and controls (3 groups). ${ }^{4}=$ AT is statistically significant different between patient group $>0.2$ and controls ( 3 groups). Error bars represent SEM.

Figure 5. The relationship between Clinical Oral Dryness Score (CODS) and Unstimulated Whole salivary flow rate (UWS) of dry mouth patients and healthy subjects (aged matched controls). Patients in the lowest UWS flow rate group have the highest CODS. The CODS was significantly $(p<0.01)$ increased in all three patients groups compared with the controls.

Keys:

${ }^{1}=$ significantly different in all three patients groups compared with the controls.

${ }^{2}=$ statistically significant between patients group $0-0.1$ and $>0.1-0.2 \mathrm{ml} / \mathrm{min}$.

${ }^{3}=$ statistically significant between $0-0.1$ and $>0.2-0.3 \mathrm{ml} / \mathrm{min}$. Error bars represent SEM. 

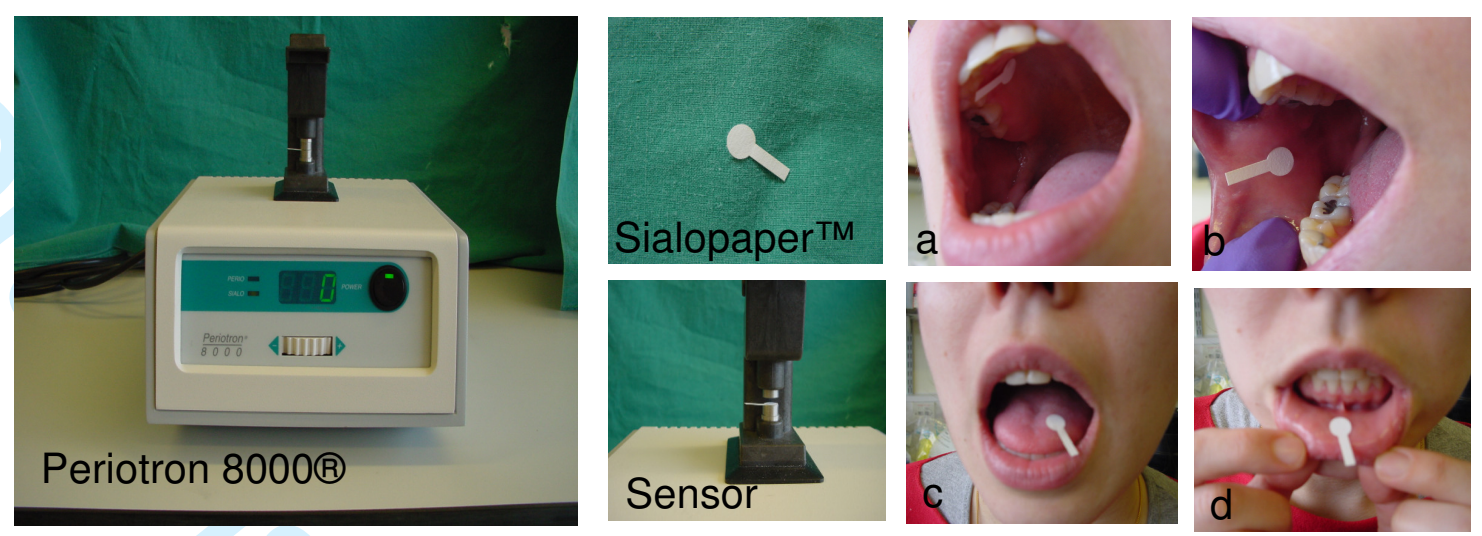


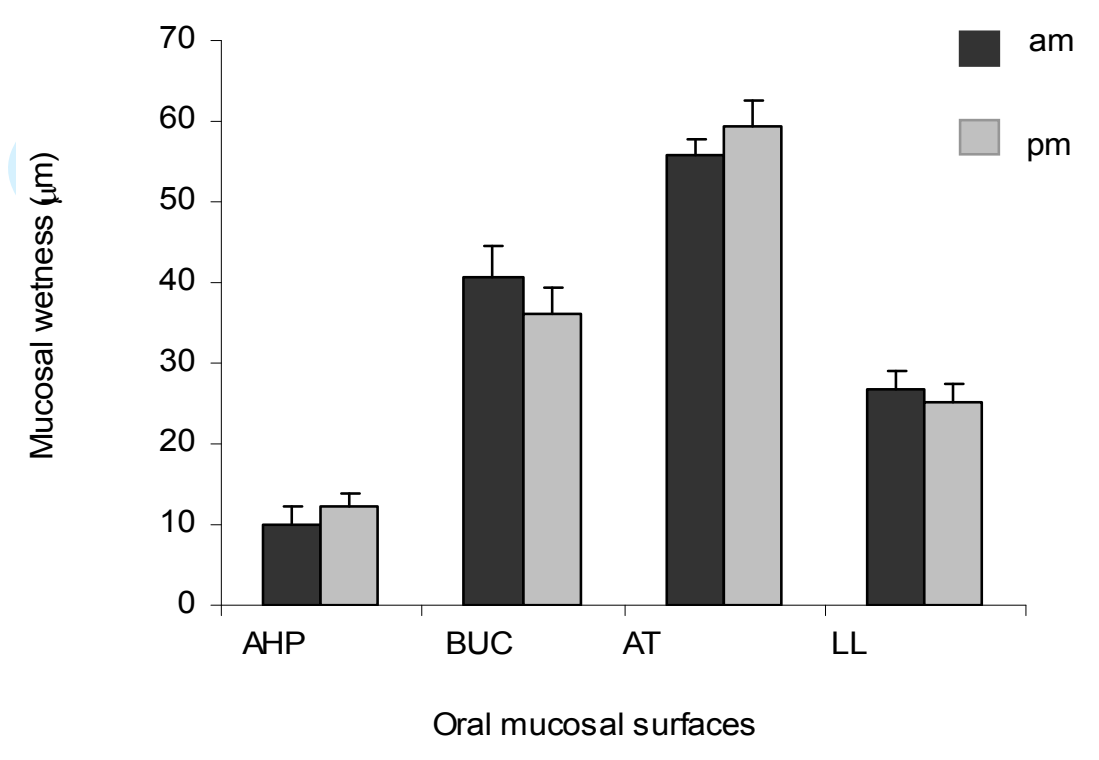

Figure2

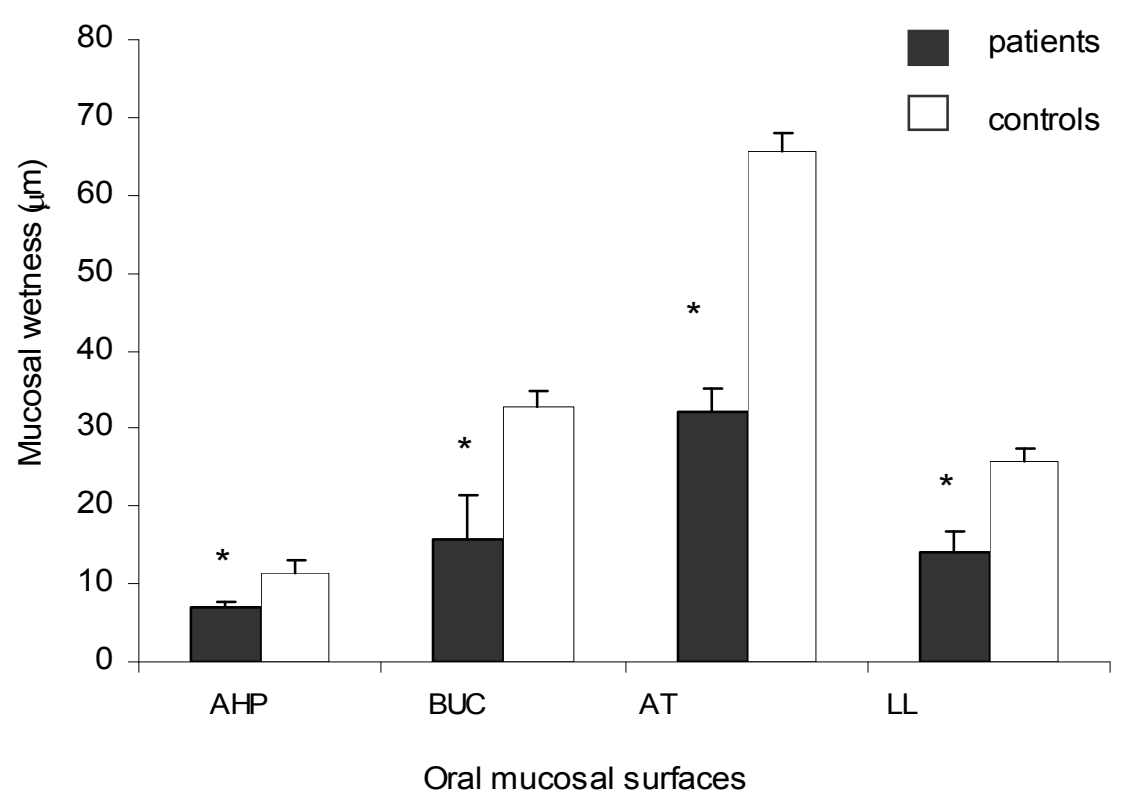

Figure3 

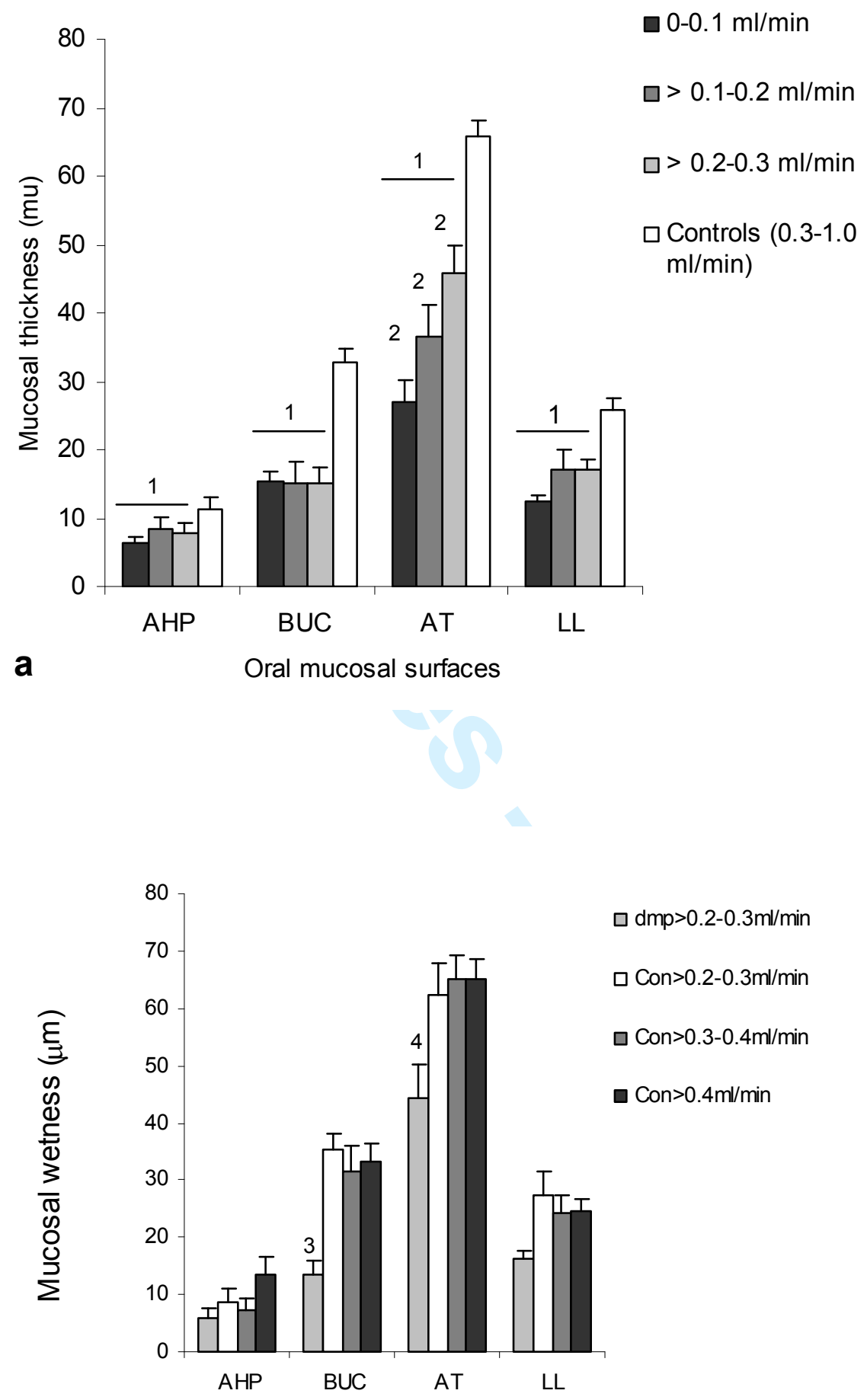

b Oral mucosal surfaces

Figure4a

Figure 4b 


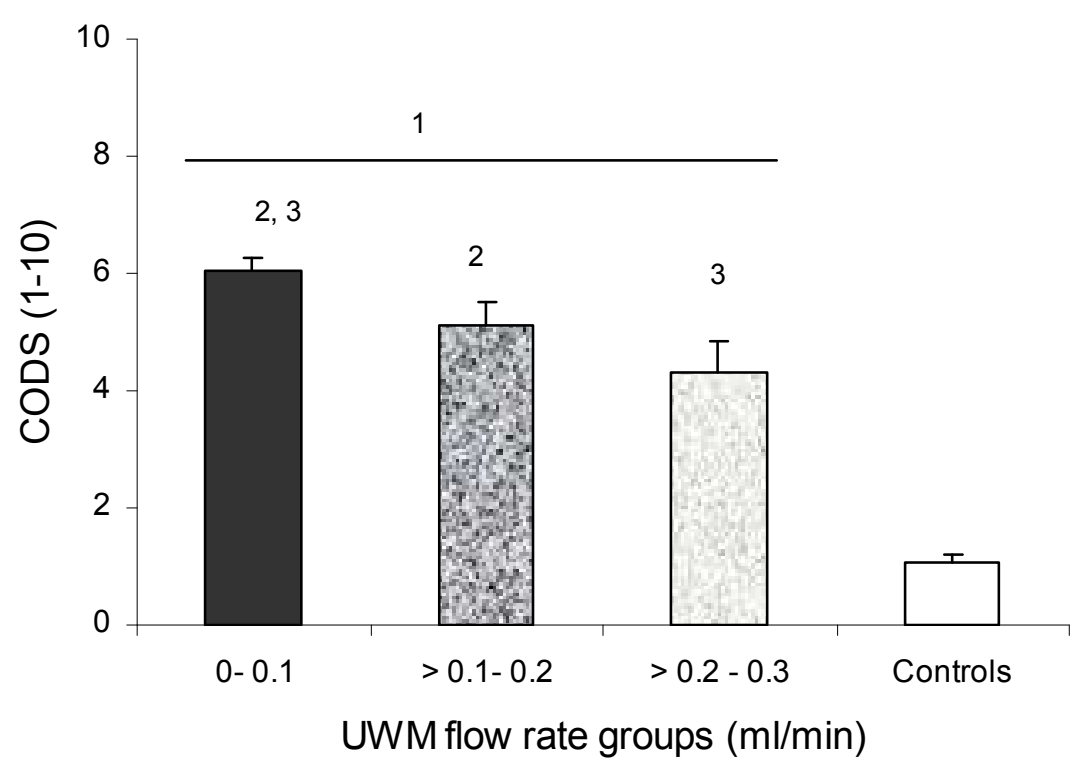

Figure5 
$1-$ April-2010

Dear Prof Baum

Manuscript ODI-08-09-OM-1372.R2, entitled "Investigating the relationship between hyposalivation and mucosal wetness", which you submitted to Oral Diseases, has now been change according to the reviewers' comments

Kind regards

Yours sincerely,

Dr Samira Osailan

Reviewers' Comments to Author:

Reviewer: 1

The paper has further improved. However, the authors still fail to be consistent with the nomenclature:



Response: The word years was added

- Page 6, line 41/42: either use $<0.1 \mathrm{ml} / \mathrm{min}$ (bold

first time it is use as was done for 0.1-0.2 and >0.2) or 0-0.1 and >0.1-0.2 and >0.2. Now still the manuscript and figures are not similar. E.g., to what group belongs 0.1 ? Response: page 9 line $41 / 42$, it is changed to $0-0.1$ - References: please apply the instructions to the authors. Now the abbreviations of the journals are not always correct, e.g. J Den Res in stead of J Dent Res, Archs oral Biol in stead of Archs Oral Biol as well as that dots are added to the abbreviations of the journals e.g. J. Dent. Res. at some spot and not at other spots. Please review carefully the instructions to the authors. Response: References were checked and changed according to the reviewer's recommendation

- Add the volume number and page number to the reference of Challacombe in $J$ Dent Res.

Response: this is an abstract publication (http://iadr.confex.com/iadr/2008Toronto/techprogram/abstra ct_106439.htm) 
Reviewer: 2

I strongly recommend not using the term Unstimulated Whole Mouth salivary flow rate as it is very rare in the literature. Using sometimes UWM salivary flow rate, on other occasions UWM flow, on others UWM flow rate and still on others solely UWM for the same concept is confusing. It will be much simpler and straight forward to use Unstimulated Whole Salivary Flow Rate and the abbreviation UWS throughout the entire paper.

Response: Unstimulated Whole Mouth salivary flow rate is now change to Unstimulated Whole Salivary Flow Rate with abbreviation UWS throughout the whole paper as recommended.

Associate Editor:

Please use consistent nomenclature throughout the paper. The AE urges strongly the use of unstimulated whole saliva (UWS) or unstimulated whole salivary flow rate in the manuscript.

Response: UWS abbreviation is now used for unstimulated whole salivary flow rate through out the whole paper 